\title{
EIN JAHR EUROPAGEOGRAPHIE \\ AN EINER BEZIRKS- ODER REALSCHULE
}

\author{
MAX H. DISTELI
}

\section{EINFÜHRUNG UND VORAUSSETZUNGEN}

An den aargauischen Bezirksschulen wird die Behandlung Europas der zweiten Klasse (7. Schuljahr) zugewiesen. Die Geographie ist von der ersten bis zur vierten Klasse durchwegs mit 2 Wochenstunden vertreien. Europa soll im Anschluß an die Besprechung der Schweiz, für die ebenfalls ein Jahr vorgesehen ist, behandelt werden. Eine größere Zahl von Grundbegriffen soll vorhanden sein. Der Schüler ist mit der Karte so weit vertraut, daß er Tiefland und Hochland, Gebirgsketten erkennt, die Richtung von Flüssen anzugeben vermag, mit dem Maßstab umzugeben weiß, Links und Rechts, Oben und Unten zu unterscheiden vermag und die Himmelsrichtungen auf der Karte richtig weisen kann. Er vermag ferner die wichtigsten Signaturen zu deuten, findet unter anderem breite Täler und Schluchten, Südabhänge und Nordabhänge, Sonnseite und Schattseite, Längstäler und Quertäler.

\section{DIE ZIELE EINES EINJÄHRIGEN EUROPAGEOGRAPHIE-UNTERRICHTES}

Es besteht die Gefahr, daß wir sie zu hoch schrauben und damit verfehlen: Wir wollen zuviel behandeln und dies zu vollständig, wir verwenden Begriffe unter denen sich der Schüler nichts Exaktes vorstellen kann (wie zum Beispiel Industrie, Landwirtschaft, Klima) dann und wann haben wir überhaupt kein Ziel und schweifen in Nebensächliches ab, weil wir nicht wissen, was Geographie eigentlich soll. Wir zeigen zu viele Bilder, besonders über Gebiete, die wir selber bereist haben. Wir verlieren uns in der Aufzählung von Produkten und Statistiken, deren Zahlen doch nicht stimmen und meist überhaupt keinen Bildungswert besitzen. Wir können ein Ziel nur ins Auge fassen, wenn wir die Möglichkeiten, die wir haben, streng berücksichtigen. Das erste, wozu wir uns aufraffen müssen, ist der Mut zur Lücke, zum Weglassen. Das zweite ist, daß wir wissen, was wir wollen. Dabei kommen wir nicht um die Frage herum: «Worin besteht das Geographische?» Es besteht im Verbinden von Einzelheiten zu einer umfassenderen Gesamtheit, der Landschaft. Was wir dann behandeln, ist nun weniger wichtig als wie wir es behandeln; dies ist entscheidend und kann das Interesse fördern oder zum Erlahmen bringen. Welche Möglichkeiten wir immer in der Stundenzahl haben, das Ziel muß sein: den Schüler Kartenlesen lehren im weitesten Sinne. Das will gehörig geübt sein, gibt aber den Schlüssel für die Türe zum Verstehen der Landschaft. Nicht nur lernt der Schüler dabei genau beobachten, er lernt auch kombinieren und damit Zusammenhänge sehen. Der Sekundarschulatlas ist, richtig ausgewertet, ein erstklassiges Bildungsmittel. Allerdings muß der Lehrer nun die verschiedenartigsten Methoden anwenden, um ihn immer wieder «schmackhaft» zu machen: Wandbild, Lichtbild, Namen von Spezialitäten aus europäischen Ländern usw. Dem abstrakten Kartenbild muß auf mannigfaltigste Art «Leben» eingehaucht werden.

\section{MÖGLICHKEITEN WÄHREND EINES JAHRES}

40 Schulwochen ergeben 80 Lektionsstunden, wovon wir 10 wegen Ausfalles abziehen müssen. Es bleiben 70 Stunden zur Verfügung, die wir vielleicht ganz verschieden aufteilen können. Für die etwa 25 Staaten würden noch nicht einmal je 3 Stunden zur Verfügung stehen. Es hätte wohl wenig Sinn nach dieser zu nichts führenden Vollständigkeit vorgehen zu wollen, ebenso abwegig, wie wenn wir sämtliche Tiere oder alle Pflanzen besprechen möchten. 
Wir wollen jetzt einmal einen Jahresplan gestalten, um das Ziel, das wir gesteckt haben, einigermaßen zu erreichen. Gerade, weil wir nur ausgewählte Länder zu besprechen beabsichtigen, reservieren wir einmal 20 Stunden für den ganzen Erdteil, wobei wir den komplizierten Umriß viele Male zeichnen lassen, die Halbinseln und Inseln nennen, die starke Verzahnung mit dem Meer hervorheben und Größenvergleiche mit der Schweiz anstellen. Dann gehen wir zum Beispiel über zu Tiefländern und Hochländern, zu den wichtigsten Gebirgsketten und zu den Flußtälern von ganz Europa. Schon jetzt müssen die Spezialkarten herangezogen werden, und die Schüler haben herauszufinden, wo Europa feucht ist, wo trocken, wo im Winter kalt und im Sommer heiß, wo im Winter mild und im Sommer relativ kühl. Einige Meere frieren zu, andere nicht. Norwegens Küste führt uns zur Warmwasserheizung des Golfstromes. Auch die Länder werden aufgezählt, unterschieden in Binnenländer und Küstenländer. Hauptstädte werden mit den Flüssen, an denen sie liegen, genannt oder mit den Meeren, an welche sie grenzen. Die wichtigsten Völker des Erdteils, ihr Aussehen und Beispiele ihrer Sprache (kann ersetzt werden durch Volkslieder oder durch Volksmusik) sollen den allgemeinen Teil beschließen. Dabei lernen die Schüler verschiedenartige Atlaskarten aufsuchen und benutzen, und sie erfassen, was der Atlas alles bietet. Es verbleiben 50 Stunden für die Länder. Da wollen wir jetzt das Schwergewicht auf unsere Nachbarländer legen, die jeder Schüler genauer kennen lernen sollte. Wählen wir für Deutschland 10 Stunden, so können wir aus der Vielgestalt dieses Landes Weniges auswählen, das grundsätzlichen geographischen Bildungswert besitzt, wie Gezeiten, Marschland (Erzählung Sturmnacht auf einer Hallig einflechten), Nehrung, Dünen, Trichtermündung, Haff. Es läßt sich das Wesentliche über Holland gerade damit besprechen. Thema: Der Mensch und das Meer. Nachdem wir ein möglichst lebendiges Bild des norddeutschen Tieflandes und ein paar klare Begriffe geschaffen haben, drängt sich die Behandlung etwa des Rheintals von Basel bis zur Mündung auf. Da erarbeiten wir einige klare Vorstellungen über Größe und Entstehung dieses Grabens, über die Trockenheit dieses Gebietes, den Städtereichtum, der mit der Fruchtbarkeit des Bodens zusammenhängt, über den einstigen herrlichen Rhein und über den verschmutzten, kanalisierten Rhein von heute. Über das Wasser muß gesprochen werden, nicht über den Standort irgendwelcher unbedeutender Industrien. Schon auf dieser Stufe ist nichts wichtiger als die natürlichen Grundlagen und ihr Zusammenwirken. Bei Baden-Baden können wir auf Bekanntes in unserem Lande zurückschließen und fruchtbar repetieren. In gebotener Kürze behandeln wir den malerischen Durchbruch des Rheins durch's Schiefergebirge, die rebentragenden Sonnenhänge, schildern und zeigen den regen Schiffsverkehr. Die gefährlichen Stromschnellen müssen erklärt werden und dabei auch das Lied von der Loreley. Mit dem Eintritt in die Tieflandsbucht von Köln wandelt sich das Bild grundlegend. Jetzt wollen wir einmal erzählen, wie es in einer Industriesteppe aussieht und warum gerade an dieser Stelle eine solche besteht. Dabei muß uns zunächst wieder der Atlas helfen. Die Schüler sollen eine Vorstellung von einem Kohlenbergwerk bekommen, sie sollen sehen, was ein Hochofen leistet und womit er beschickt wird. Da soll das Begriffliche bei der lebhaften Schilderung dieser technisierten Landschaft ganz in den Hintergrund treten. Vater Rhein ist alt und krank geworden. Jetzt sollten wir noch einmal zu seinem Ursprung zurückkehren, wo das Wasser klar und behende dahinfließt. Ich glaube, daß es verfrüht ist, von EWG und Montanunion zu sprechen. Das soll dem reiferen Alter vorbehalten bleiben. Ein Bezirksschüler kann die Tragweite dieser Zusammenschlüsse noch nicht erfassen.

Um auch Österreich einbeziehen zu können, behandle ich als dritte Landschaft noch die Schwäbisch-Bayrische Hochebene, zu welcher der Schüler den Anschluß vom Schweizerischen Mittelland aus recht gut finde. Es gibt auch sonst noch einige Parallelen wie die Alpenrandseen, die Moränen und die Schotter, Sande und Lehme, die trockene und moorige Regionen bewirken. In einem andern Jahr können wir weitere Landschaften Deutschlands in den Vordergrund rücken, so den Schwarzwald, 
den Fränkischen Jura, die Mecklenburger Seenplatte, die Steilküste Rügens, je nach Anschauungsmaterial und geographischer Ergiebigkeit. Pragmatische Gesichtspunkte sollen nicht entscheiden. Wir wollen ohne irgendwelchen praktischen Nutzen unterrichten, weder im Hinblick auf Post, auf Bahn oder ähnliche Berufsbedürfnisse, weil das den Blick kanalisiert. Nichts bereitet besser vor als eine gute theoretische Bildung, und ein ausgiebiges Fördern der Gemütswerte der jungen Menschen. Eine kurze Zusammenstellung der zu arbeitenden Begriffe (ohne ein Minimum von Namen) ergibt etwa:

Tiefebene, Flachküste, Gezeiten, Brandung, Watt, Marsch, Polder, Deich, Geest, Nehrung, Düne, Luv, Lee, Haff, Schelfmeer, Mündungstrichter, Landwind, Seewind, Bucht, Graben, Stromschnellen, Durchbruch, Flußkorrektion, Wasserstraße, Industrielandschaft, Rauchglocke, Kohlenbergwerk, Flöz, Kumpel, Bewetterung, Hochofen, Abraum, Erz, Koks, Roheisen. Hochebene, Alpenvorland, Alpenrandsee, Moräne, Schotter, Mergel und Ton, Moor, Grundwasserspiegel, Verkehrsknoten, Löß, Alpenstaat, Ostalpen, Becken.

Bei der Besprechung Italiens, dem wir auch 8 Stunden einräumen wollen, könnten wir einmal von den verschiedenen bekannten Produkten ausgehen, die wir nennen lassen: Orangen, Zitronen, Pfirsiche, Trauben (Wein), Gemüse, Tomaten, Salami, Mortadella, Spaghetti, Risotto, Polenta. Sicher werden auch genannt: Fiat, Lancia, Vespa, Lambretta. Daraus soll Geographie werden, das heißt, wir müssen zur Landschaft vordringen. Vielleicht etwa so: Salami - Schweinefleisch - Schweine - Schweinefutter Gerste - Mais. Spaghetti - Weizenmehl - Weizen - Weizenanbau, Tomatenpürée Tomaten - Tomatengroßanbau. Risotto Milanese - Reiskorn - Reispflanze - Reisanbau. Es muß fruchtbar sein, wo diese Kulturpflanzen in Menge wachsen. Wir möchten wissen, wie es dort aussieht. So kommen wir ungezwungen wieder zu Wand-und Atlaskarte, die uns darüber belehren. Wie weit wir hier die Poebene besprechen, ist unerheblich, die Hauptsache ist, daß wir ein paar zusammenhängende Fakten bis zur landschaftlichen Wirklichkeit hin verfolgen. Den Begriff «Halbinsel» können wir bei Italien sehr schön herausarbeiten. Der jährliche Verlauf der Niederschläge, das Zuviel und Zuwenig, das durch die Entwaldung stark gesteigert worden ist, wird am besten geschildert. Es können Zeitungsausschnitte von Überschwemmungskatastrophen verwendet werden. Der Stiefel: Ein Land ohne Wald, daher das Land der unberechenbaren Flüsse. Als feuerspeienden Berg wählen wir den Vesuv, den Aetna oder den Stromboli zur Begriffsbildung aus. Auf der Karte suchen wir Kegelberge, die einst tätig waren, heute aber ruhig sind. Bilder von Oelbaum-, von Orangen- und Zitronenhainen, von $Z_{y}$ pressenund Pinienwäldchen und von den Pflanzen selber beschließen das Kapitel Italien. Viele Zeichnungen werden vom Lehrer an die Tafel gezeichnet und von den Schü!ern ins Heft. Die Zeichnungen werden nicht polykopiert abgegeben. Ein Lehrbuch kann u. U. entbehrt werden. Es verleitet gern zur Schablone. Wir bekommen jedes Jahr auf anderem Wege an andern Stoff heran, der ebensogut geographisch sein kann.

Zusammenstellung der Begriffe: Aufgefüllte Meeresbucht, Delta, eingedämmter Fluß, Garten Europas, Alpenflüsse, Apenninenflüsse, Lagune, Lido (Repetition zu Haff und Nehrung), Pfahlsiedlung, Alpenrandseen (Vergleich mit den nördlichen), Bewässerungskanäle, Mischkultur, Nahrungsmittelindustrie, Leichtindustrie, Mittelmeerklima, Halbinsel, Wassernot, Wassersnot, Vulkan, Krater, Asche, Lava, Schlot, Dampfpinie, Kegelform, Olivenbaum, Südfrüchte oder Agrumen, Pinien und Zypressen. Bodenverwüstung. Warenaustausch, Gütergroßverkehr durch die Schweiz und über den Brenner.

Frankreich schließt sich an: Wir sollten auch da von etwas ausgehen, das uns etwas Weniges von diesem großen Lande zugänglich macht und des Schülers Interesse weckt. Gehen wir einmal von Paris, der Hauptstadt, aus! Wir möchten von uns aus nach Paris reisen und den Zug benützen! Welche Möglichkeiten stehen uns zur Verfügung? Wir wollen nach der Karte den Weg beschreiben. Es werden einige Orte genannt und richtig ausgesprochen, dann Höhenzahlen bis und mit Paris gesucht. Dasselbe machen wir 
auf einer Zufahrt von Deutschland her. So können wir den Beckenbegriff vorbereiten. Wir messen auch Distanzen, und die Schüler sollen darüber sprechen, wie die Bahnlinien auf Paris zulaufen. Alles in Frankreich richtet sich nach Paris. Diese Stadt verschlingt Riesiges, weil sie ein Riese ist. Wie verlaufen die Bergrücken im Osten, Südosten und Süden der Stadt? Was für eine Küste bildet Frankreich am Kanal? Wir zeigen Bilder davon. Welch ein Unterschied zur norddeutschen Flachküste! Jetzt wollen wir die Pariser Gegend modellieren (z. B. Sandkasten oder Karton). Wir können es auch mit Tellern versuchen, am besten mit solchen verschiedener Farben. Die harten Schichten bilden die Steilhänge und die Falaiseküste, die weichen sind stark ausgewawaschen und bilden flache Gebiete. Über Paris wird erzählt, und es werden einige Begriffe anschaulich und lebendig vorgestellt und dazu Bilder demonstriert. Welcher Bahnweg ist der schnellste von Paris nach Marseille? Finden wir in der Schweiz etwas mit dem Massif Central in Frankreich Vergleichbares? Welche Form hat dieser Gebirgsstock? Größe vergleichen mit der Schweiz! Wir zeigen einige Bilder über die Auvergne und lassen nach den Bergformen auf die Entstehung raten. Welchen Weg wählt ein Franzose von Straßburg nach Nizza, welchen Flüssen und Senken folgt er und was sieht er als aufmerksamer Reisender unterwegs? Wir messen die Distanzen Basel-Paris und Paris-Brest. Wir messen auch Marseille-Calais. Worin besteht ein wichtiger Unterschied zwischen Italien und Frankreich? Frankreich grenzt z. B. ans Atlantische Meer und zugleich ans Mittelmeer. Wo finden wir Trichtermündungen, wo Nehrungen und Dünen? (Weltstadt, Boulevard, Place de l'Etoile, Champs-Elysés, Metro, Polypenstadt, Mansarden.)

Steilküste, Becken, artesisches Wasser, Mondlandschaft im Massif Central, Côtes, Mistral, Languedoc, Languedoui, ertrunkene Täler, Burgunder Pforte, Burgundische Senke, Ceinture d'orée, Côte d'or, Champagne pouilleuse, Champagne humide, Weinbaugrenze. Mit 8 Stunden sollten wir durchkommen, wobei wir auch früher erarbeitete Begriffe repetieren können.

Gehen wir nun zu einem Inselreich über, zu Großbritannien. Da sollten wir mit 6 Stunden rechnen. Der Schwierigkeit der Aussprache der Namen Rechnung tragend, würden wir eine Stunde nur Namenkunde betreiben und die Namen richtig aussprechen lernen. Mit der Gruppierung der wichtigsten Namen nach Ortschaften, Gebirgen, Flüssen, Meeresbuchten und dem Zeigen auf der Karte vergeht eine weitere Lektion. (Lit. Schulpraxis, Monatsschrift des Bernischen Lehrervereins, April 60, Heft 1, Max Wehrli: Aussprache fremdländischer geographischer Namen, Bern.) Welche Lektionen wollen wir aus dem gewaltigen Stoff herausschälen? Wir besprechen am besten das physische Kartenbild Seite 42 im Atlas, dann auch Seite 46, wo Europa als Ganzes dargestellt ist und wo der Landsockel um die Inseln besonders deutlich zutage tritt. Die Volksdichtekarte muß uns zur Frage führen, wovon die britische Bevölkerung lebt. Auf die Flachsee und die Fischbänke können wir näher eingehen. Begriffe: Insel, Halbinsel, Schelfmeer, Bank, Firth, Golfstrom, Smog, Parklandschaft, Dock, Commonwealth.

Skandinavien, für das wir auch 6 Lektionen verwenden wollen, können wir zunächst als Ganzes in seiner eigenartigen Form ansprechen, die zu Vergleichen und zum Nachzeichnen anregt. Nach der Karte Seite 40 im Atlas müssen wir zuerst erklären, was wir unter dem Wort Skandinavien verstehen. Wir lassen dann die gewaltige Länge in nord-südlicher Richtung messen und vergleichen mit Distanzen im bereits behandelten Gebiet Europas. Wir erkennen Brücken, die von Mitteleuropa nach den Ländern Skandinaviens führen. Bis wohin können wir von Zürich aus mit direkten Eisenbahnwagen nach Skandinavien fahren? Wir lassen diese Reise mit wichtigen Zwischenorten beschreiben und kommen auf die Eisenbahnfähren, die an bestimmten Orten konzentriert sind. Für Norwegen würden wir uns auf die Besprechung der Fjorde, auf den Regenreichtum und die Milde seiner Küste beschränken, in Schweden auf die winterliche Kälte im Gegensatz dazu (Karte Seite 48 Januar-Isothermen), dann auf den Be- 
griff Schärenküste, auf den Fluß- und Seereichtum und die Lage der Wasserscheide. Finnland stellen wir als das Land der tausend Seen und der unendlichen Wälder vor. Bilder über die Mitternachtssonne und die vom Polarlicht erhellte Polarnacht müssen das Bild von Skandinavien beschließen. Das Wirtschaftliche besprechen zu wollen, führt leicht ins Uferlose. Wir lassen es lieber weg; aber einige wenige Begriffe müssen klar herausgearbeitet werden: Halbinsel, Kap, Fjord, Mitternachtssonne, Polarnacht (die beiden letzten ohne Erklärung) Schärenküste, der Lappländer, Seenplatte und Vergletscherung, Eisenbahnfähre, Vogelfluglinie.

Wir kehren in den Süden Europas zurück und sehen uns den Kopf der Europafigur, die Pyrenäenhalbinsel, in 4 Lektionen an. Da können wir an die Eigenart des Mittelmeerklimas anknüpfen, wie wir es beim Stiefel schon besprochen haben. Wir können die Gemeinsamkeiten mit der Apenninenhalbinsel und die Besonderheiten der Iberischen Halbinsel hervorheben. Wir lassen jetzt auch die wichtigsten Namen wieder repetieren und an der Wandkarte zeigen. In welcher Richtung fließen die verschiedenen Flüsse? In welcher Richtung verlaufen die Gebirge? Die Lage der beiden Hauptstädte werden verglichen. Wir wollen die Meereshöhe der verschiedenen Hauptstädte Europas suchen. Welche liegt am höchsten? Wohin schaut Portugal ausschließlich, wohin Spanien? Beide hängen nur mit einer Landseite am übrigen Europa. In Spanien spürt man schon den Hauch Afrikas. Wie heißt der südlichste Punkt des Festlandes von Europa? Iberien, ein Land, wo das Wichtigste fehlt: das Wasser.

Begriffe: Hochland, Randland, Mittelmeertyp des Flusses, Bewässerungsoasen, Landbrücke nach Afrika.

Es verbleiben noch 8 Stunden. Den Riesen Rußland und sein Kommunistisches System würde ich erst in der 4. Klasse behandeln, in der die Schüler solche Probleme besser zu erfassen vermögen. Ich würde einen Teil der 8 Stunden noch den Donauund Balkanländern widmen. Da ich selber Jugoslawien etwas kenne zeige ich Bilder über die Karsterscheinungen dieses Landes, erzähle von der Trostlosigkeit des Hochkarstes und seinen Ursachen, dann von dem bunten Völkergemisch und den Mohammedanern und Zigeunern. Welcher Bub möchte nicht in eine solch geheimnisvolle Höhle

wie die von Postojna oder die noch schönere von Divaca, wo in abgrundtiefen Schlünden Wasser versickert, um nach $40 \mathrm{~km}$ Lauf an die Oberfläche zu treten? Auch die Eind rücke einer Griechenlandreise können Anregungen für den Unterricht vermitteln. Die restlichen Stunden lassen sich zu verschiedensten Repetitionen, auch in Spielform, gestalten.

Damit hat die Klasse einen Überblick über Europa erhalten, der zwar nicht vollständig ist - wie könnte er das je - und hat auch einige grundlegende Begriffe von diesem einzigartigen Erdteil. Was ich hier entworfen, ist ein Versuch, Varianten des Unterrichts zu skizzieren, um die Fülle des zusammenhanglosen Aneinanderreihens zu umgehen und doch die Forderungen des Lehrplanes nach Möglichkeit zu erfüllen.

\section{LITERATUR}

Gürtler A. und Wolf L.: Zeichnen im erdkundlichen Unterricht, Verlag Ernst Wunderlich, Worms, Hefte 1, 2, 5. Verschiedene Nummern der neuen Schulpraxis, St. Gallen. Schlunegger: Die Länder der Erde für den Geographieunterricht gezeichnet.

UNE ANNÉE D'ENSEIGNEMENTS DE GÉOGRAPHIE SUR L'EUROPE A UN PROGYMNASE

L'auteur essaie de donner, sur la base de 2 leçons par semaine, un plan chronologique et un plan des sujets à étudier au cours d'une année. Comme il est impossible de traiter tous les pays avec une classe, l'Europe est d'abord traitée comme un tout, à l'aide de cartes géographiques de l'Atlas de l'école secondaire. Ensuite, des idées géographiques fondamentales sont dégaées. D'après certains pays choisis, et cela de nouveau à l'aide des cartes par une restriction considérable de la matière, en évitant le plus possible la question: A quoi ceci et cela est-il utile à l'homme? L'auteur croit rendre service à l'enseignement de la géographie. 